closer scrutiny, be reinstated and disclosed. Such a defensive response to patients having access to records is not inevitable and there are well documented examples from general practice (e.g. Baldry, 1986) and psychiatry (Essex et al, 1990) where actively encouraging patients to share the medical record has been a positive experience for both doctors and patients. Such sharing was entirely compatible with responsible medical practice and supplementary 'confidential' records were unnecessary.

Lipsitt (1980) discussed patient and doctor responses to the introduction of Chapter 214 of the Massachusetts Acts of 1979 which guaranteed patients in the USA access to their records. He noted that few patients sought access to their records despite having the right to do so, and suggested that the traditional doctor-patient relationship, modelled on the parent-child interaction, carries subtle cues which define who will control and who will be controlled. Many doctors found the idea of patients having access to their records threatening to the balance of this relationship and label those who break the taboo and ask to see their records as deviant. Lipsitt warned that attitudes and traditions in medical care are more likely to be modified by an awareness of patients and their feelings than by an incantation of laws.

Gaitonde underplays the potential benefits to patients of the new legislation. Ellis (1979) showed that giving medical patients written information about their conditions significantly improved understanding and recall. An efficient way of providing such information is in the form of a patient-held record which the doctor writes in and the patient keeps. Essex et al (1990) have reported the use of such a record with psychiatric patients receiving care shared between general practice and out-patients. They found that the record was enthusiastically accepted by patients who valued being consulted and thought they were in a better position to challenge their doctor. The record also improved communication between staff. Despite the obvious benefits of the system, these authors also reported a negative response by most psychiatrists and nurse managers consulted.

Patients today expect more information and a more collaborative working arrangement with their doctor. This trend is reflected in the current legislation and is likely to grow in the future. The profession can react to this trend either defensively to maintain the traditional paternalistic approach to patients, or by viewing it as a way of forging a closer relationship with the patient and adding it to their therapeutic armoury. If the latter is to apply, doctors will have to learn how to write records in a manner which patients will understand, and record information which is useful to the patient as well as to professionals. Rather than inhibiting record keeping this would result in an enriched record and improved patient care. Teaching doctors how to write such records should become an important element in undergraduate and postgraduate education and offers a particular challenge to psychiatry. It is a challenge which should be taken up sooner rather than later.

Baldry, M., Cheal, C., Fisher, B. el al (1986) Giving patients their own records in general practice: experience of patients and staff. British Medical Journal, 292, 596-597.

Ellis, D. A., Hopkin, J. M., et al (1979) "Doctors' orders": controlled trial of supplementary, written information for patients. British Medical Journal, Feb 17, 456.

EsseX, B., DoIG,R. \& RENSHAW, J. (1990) Pilot study of shared care for people with mental illnesses. British Medical Journal, 300, 1442-1446.

JoNes, R. B., Hedley, A. J., AlLISON, S. P., et al (1988) Censoring of patient-held records by doctors. Journal of the Royal College of General Practitioners, 38, 117-118.

LIPSITT, D. R. (1980) The patient and the record. New England Journal of Medicine, Jan 17, 168-169.

Department of Psychiatry

Paul Mclaren

Guy's Hospital

London SEI 9RT

\section{Anorexia nervosa in Asian children}

SIR: Bryant-Waugh \& Lask (Journal, February 1991, $158,229-233$ ) have reported cases of anorexia nervosa (AN) in Asian children which, although interesting, are not the first, as Badrinath (Journal, April 1990, 156, 565-568) and Neki et al (1977) have also reported similar cases.

The earlier report by Badrinath has generated a lively discussion to which we would like to add some of our own experiences with AN in India.

Although AN may be less common in India than in the West, it is not as rare as these reports suggest. Indeed, a likely explanation for the absence of reports in literature is the familiarity of the diagnosis rather than its rarity.

At our general hospital psychiatry department, we have encountered five new cases of $A N$ in the last four years. With the average annual intake of about 2500 new patients, the frequency of presentation is quite similar to that reported by Buhrich (1981) in Malaysia. All were unmarried females aged between 13 and 25 years. Contrary to the experience of Khandelwal \& Saxena (Journal, November 1990, 157,784 ), three of our patients did have body image disturbance while none had associated hysterical symptoms. 
Much of the discussion on AN in Asians has focused on the role of exposure to Western ideas and culture which emphasise slimness and diet control. However, three of our patients came from very traditional Indian households. The youngest patient had never been sent to school by her illiterate parents and had not even travelled outside her village until she was forced to do so because of her symptoms. The view that slimness is not a requisite for beauty in Indian culture may also be an oversimplification. Anything beyond a well-fed look in an adolescent is very likely to subject her to ridicule from peers and family members (a precipitating factor in three of our patients), and may adversely affect her prospects even in the arranged marriage system.

Our observations seem to indicate that conflicts over body weight may not be a phenomenon unique to the West. The lower prevalence of AN in India may be because of the qualitative and quantitative aspects of the diet in a less affluent society, the tradition for women to eat the last, and often the least, in the family, and the society's acceptance of a wider range of weight as being consistent with good appearance. Consequently, fewer women would cross the upper limit and feel under pressure to diet.

Buhrich, N. (1981) Frequency of presentation of anorexia in Malaysia. Australian and New Zealand Journal of Psychiatry, 15, 153-155.

Nekı, J. S., Mohan, D. \& SoOd, R. K. (1977) Anorexia nervosa in a monozygotic twin pair. Journal of Indian Medical Association, 68, 98-100.

Department of Psychiatry

D. H. GANDHI

M. Prakash Appaya Tanya Machado

St John's Medical College

Bangalore 560034

India

The clinical application of restricted environmental stimulation therapy (REST): observations of a psychiatrist

SIR: The idea that solitude and the reduction of environmental stimulation are conducive to the improvement of mental health, and health in general, is not new. What is new is the development of a precise technique by which environmental stimulation can be reduced and controlled. The exact methods employed in the REST programme have been described elsewhere (Suedfeld, 1980).

The REST programme is the result of a pioneering research by Suedfeld and his colleagues at the department of psychology at the University of British Columbia.
Its origin lies in the massive research done by Zubek and his colleagues in the 1960s in the area of sensory deprivation. The initial data on the subject have been summarised by the late Professor Zubek (Zubek, 1969). REST uses several of the major concepts of the sensory deprivation technique, but departs from it by abandoning, "physical restraints, cardboard cuffs, gloves, goggles and ear-phones etc. ..." (Suedfeld \& Kristeller, 1982).

The author, a practising psychiatrist in Vancouver, Canada, was impressed with the positive results obtained in the treatment of tobacco addiction. One of his employees, a senior health care nurse, was a heavy smoker. The nurse had tried various methods to stop smoking cigarettes. Finally, he volunteered to undergo REST treatment in a non-university setting. At that time, the REST treatment programme was in its early stage of development at the University of British Columbia.

After the completion of the programme, the nurse stopped smoking completely, and showed several positive attitudinal changes which were noted by his family members and colleagues. This case has been reported as "Mr F" in a paper illustrating the beneficial side-effects of REST in a smoking cessation programe (Suedfeld \& Best, 1977).

REST has been applied to various clinical conditions which range from obesity to autism (e.g., Suedfeld \& Schwartz, 1983). There has been some study of the effect of the REST programme in the treatment of essential hypertension (Fine \& Turner, 1982).

During the last few years, the author has referred a number of patients to the REST clinic at the University of British Columbia. These patients were randomly chosen to participate in a research project at the university which was evaluating the effect of REST programme in reducing blood pressure without the use of medication. The patients were attending my office for psychotherapy. The complaints of the patients were psychosomatic in nature; increased blood pressure was one of their symptoms. Unfortunately, these patients were not showing a great deal of improvement. Some of them had developed insight, but they were unable to change their counterproductive attitudes. The author noted remarkable changes in their attitudes, after only one session of the REST programme, which employed a flotation tank, various relaxation tapes and the REST chamber for 24 hours. The following case history of one such patient is presented as an example.

Case report. Mr PG is a 36-year-old male, East Indian, real estate agent, who was referred to the author with a history of unexplained muscular tension, neck pain, and general symptoms of anxiety. During the interview, the author 